\section{Unter welchen Voraussetzungen wählen Sie eine intermittierende Androgenblockade zur Behandlung des Prostatakarzinoms?}

Die Frage, ob und wann eine intermittierende Androgenblockade indiziert ist, verunsichert noch immer viele Urologen - zu diffizil ist die bisherige Datenlage. Umso wichtiger sind persönliche Interpretationen und Erfahrungswerte. Zusammen mit aktuellen Studiendaten lässt sich dann eine erste praktische Empfehlung geben.

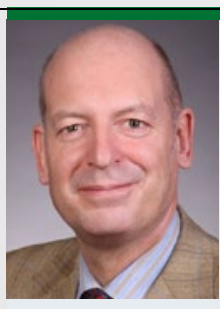

Diese Frage beantwortet für Sie

Prof. Dr. med. Johannes M. Wolff

Abteilung für Urologie Paracelsus-Klinik GolzFriedrich-Lau-Str. 11 40474 Düsseldorf E-Mail: Johannes.wolff@paracelsuskliniken.de

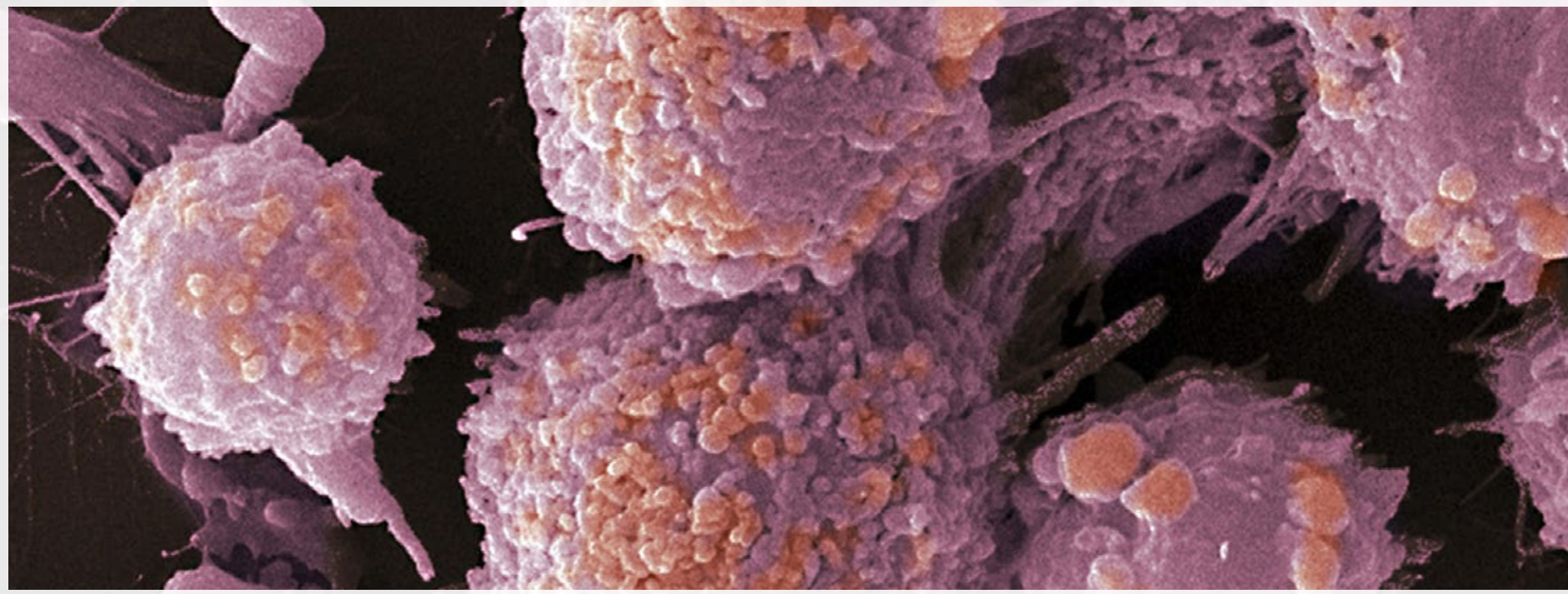

R ruchovsky und Mitarbeiter berichteten 1990 erstmals über tierexperimentelle Studien zur intermittierenden Androgenblockade [1]. Anhand eines Mausmodells wiesen sie nach, dass ein intermittierender Testosteronentzug beim Shionogi-Tumor - einem androgenabhängigen Mammakarzinom - die Zeit bis zur Androgenresistenz um das Vierfache verlängert. Seither wurde das Konzept in einer Vielzahl von Phase-2- und nachfolgend Phase-3-Studien untersucht. Neben der Möglichkeit, die Zeit bis zur Kastrationsresistenz zu verlängern, erschien für Ärzte und Patienten die postulierte Verbesserung der Lebensqualität durch die Phasen der intermittierenden Testosteronnormalisierung hochattraktiv. Obwohl in den Studien gezeigt wurde, dass es sich in der Tat um eine intermittierende Androgenblockade mit Phasen der Testosteronnormalisierung handelt, war die Verbesserung der Lebensqualität, die mit validierten Fragebögen evaluiert wurde, allenfalls marginal. In einigen Phase-3-Studien verlängerte sich zwar die Zeit bis sich eine Kastrationsresistenz entwickelte, jedoch war das Gesamtüberleben statistisch nicht signifikant unterschiedlich - obwohl es absolut in den intermittierenden Armen eher verkürzt war.

\section{Experten wagen erste Schlussfolgerung}

Auf dem jährlichen Kongress der American Society of Clinical Oncology (ASCO) im Jahr 2013 hat Maha H. Hussain, Univer- sity of Michigan, die Ergebnisse der großen SWOG-Studie zur intermittierenden Androgenblockade vorgestellt [2]. Auch hier beobachteten die Wissenschaftler, dass sich die Überlebenszeit im intermittierenden Behandlungsarm nicht signifikant verkürzte. In der dem Vortrag folgenden Diskussion fasste William Oh vom Mount Sinai Hospital, New York, die Studienlage zusammen: Er schlussfolgerte, dass die intermittierende Androgenblockade beim nicht knöchern metastasierten, fortgeschrittenen Prostatakarzinom eine Therapieoption darstellt, dagegen beim knöchern metastasierten Prostatakarzinom als Standardtherapie ungeeignet ist.

\section{Persönliche Empfehlung}

Der Empfehlung von Oh schließe ich mich bezüglich der Behandlung meiner Patienten an: Sollten Patienten mit einem knöchern metastasierten Prostatakarzinom eine intermittierende Androgenblockade wünschen, so biete ich sie als individuellen Heilversuch und damit als experimentelle Therapie an. Allerdings weise ich die Patienten explizit daraufhin, dass die Therapie möglicherweise - wenn auch nur gering - die Überlebenszeit verkürzt.

\section{Literatur}

1. Bruchovsky N et al. Cancer Res. 1990;50(8):2275-82.

2. Hussain MH et al. N Engl J Med. 2013;368(14):1314-25. 